\title{
Patient-specific model of a scoliotic torso for surgical planning.
}

\author{
Rola Harmouche ${ }^{a}$ and Farida Cheriet ${ }^{a, b}$ and Hubert Labelle ${ }^{b}$ and Jean Dansereau ${ }^{a, b}$ \\ ${ }^{a}$ École Polytechnique de Montréal. 2500, chemin de Polytechnique, Montréal, H3T 1J4 \\ ${ }^{b}$ Hôpital Ste-Justine, 3175, Chemin de la Côte-Sainte-Catherine, Montréal H3T 1C5
}

\begin{abstract}
A method for the construction of a patient-specific model of a scoliotic torso for surgical planning via interpatient registration is presented. Magnetic Resonance Images (MRI) of a generic model are registered to surface topography (TP) and X-ray data of a test patient. A partial model is first obtained via thin-plate spline registration between TP and X-ray data of the test patient. The MRIs from the generic model are then fit into the test patient using articulated model registration between the vertebrae of the generic model's MRIs in prone position and the test patient's X-rays in standing position. A non-rigid deformation of the soft tissues is performed using a modified thin-plate spline constrained to maintain bone rigidity and to fit in the space between the vertebrae and the surface of the torso. Results show average Dice values of $0.975 \pm 0.012$ between the MRIs following inter-patient registration and the surface topography of the test patient, which is comparable to the average value of $0.976 \pm 0.009$ previously obtained following intra-patient registration. The results also show a significant improvement compared to rigid inter-patient registration. Future work includes validating the method on a larger cohort of patients and incorporating soft tissue stiffness constraints. The method developed can be used to obtain a geometric model of a patient including bone structures, soft tissues and the surface of the torso which can be incorporated in a surgical simulator in order to better predict the outcome of scoliosis surgery, even if MRI data cannot be acquired for the patient.
\end{abstract}

Keywords: Multimodal image registration, inter-patient registration, model to patient registration, scoliosis.

\section{INTRODUCTION}

Idiopathic scoliosis is a disease characterized by a complex three-dimensional curvature of the spine, manifesting itself with external deformations that are often aesthetically undesirable for patients and, in more severe cases, may affect patient functioning. ${ }^{1}$ Treatments such as bracing or surgery aim at slowing down the curvature progression or at correcting some of the undesired curvature. However, the effects of spinal correction on the external shape of the trunk cannot be reliably predicted prior to treatment completion. This is problematic for the patients that require surgery, most of them adolescents, since the external appearance of their torso is one of their primary concerns. A simulator has recently been developed ${ }^{2}$ in order to predict effect of scoliosis surgery on the external appearance of the torso. Though interesting preliminary results were obtained, the simulator lacked generalizability. This might be due to the fact that although the patients' spine and external surface were incorporated in the simulator, the different soft tissues were not specifically modeled. Simulators that would integrate a 3D model of the bones and soft tissue of the trunk could be useful in predicting surgical outcome. Such a model would require the fusion of soft tissue information, typically obtained from MRIs in prone position, spine data, typically obtained from X-rays in standing position, and trunk surface information obtained from an active vision system. However, MRIs are not routinely acquired for scoliosis patients, as the imaging equipment is costly and not always available in clinical settings. In addition, MRI acquisitions of the entire torso take a considerable amount of time, thus causing discomfort to patients that are already suffering from back pain due to their condition. In such cases, the soft tissue data can be registered to the patient's geometry from a complete generic patient model that contains bone, surface topography, and soft tissue information. This registration task is difficult for several reasons: First, there is a lack of correspondences between the image modalities being

Further author information: (Send correspondence to R. Harmouche) R. Harmouche: E-mail: rolaharmouche@polymtl.ca

Medical Imaging 2013: Image-Guided Procedures, Robotic Interventions, and Modeling, edited by David R. Holmes III, Ziv R. Yaniv, Proc. of SPIE Vol. 8671, 867120

(C) 2013 SPIE ·CCC code: $1605-7422 / 13 / \$ 18 \cdot$ doi: $10.1117 / 12.2007753$ 
registered. Second, there a mixture of both rigid and non rigid deformations that occurs between the image acquisitions, since the images are acquired at different times and in different postures (the MRIs are acquired in prone position, and the X-ray and surface topography data are obtained in standing position). Finally, the soft tissue characteristics of different patients may vary greatly depending on the person's height, weight, and spinal curvature, thus rendering the inter-patient registration of soft tissues difficult. We have previously obtained a full model of the patient's torso that incorporates bone structures, soft tissues and the external surface of the torso. ${ }^{3}$ However, inter-patient registration was not performed. Most previous inter-patient registration methods focused on the brain. These works used B-splines, ${ }^{4}$ elastic body registration, ${ }^{5}$ fluid registration, ${ }^{6-9}$ Markov random fields, ${ }^{10}$ and graph cuts. ${ }^{11,12}$ Xia et al. performed inter-patient registration on uni-modal CT data of the torso ${ }^{13}$ using intensity-based registration. Such intensity-based methods are not applicable in the context of our present work because of the lack of intensity-based correspondences between the different image modalities that we widh to register. No previous works have performed multimodal inter-patient registration of the torso.

This paper proposes a methodology for the inter-patient registration of MRIs of a generic model acquired in prone position with X-ray and TP data of a test patient acquired in standing position, while preserving the rigidity of bone structures, in order to obtain a complete 3D representation of the torso of a patient with scoliosis to be incorporated in a surgical simulator. X-ray, TP and MRI data are acquired for 2 patients with scoliosis, one of which serves as a generic model. A partial test patient model is first obtained by registering the X-ray and TP data using thin-plate splines. The spine data extracted from the MRIs of the generic model are then registered to the partial patient model by using an articulated model transformation, and the soft tissues are then confined between the surfaces of the vertebrae and the trunk using modified thin-plate spline registration. The method is tested using the Dice overlap measure. The results obtained are comparable to intra-patient registration results and show a significant improvement compared to simple rigid inter-patient registration.

\section{MATERIALS AND METHODS}

MRI, X-ray and surface topography data are acquired for the whole torso and for each of the 2 patients considered in this study (Figure 1). The patient 2 MRIs will only be used for validation purposes. Both patients have a right thoracic spinal curvature, which is hypothesised to lead to similar vertebral transformations between the MRIs in prone position and the X-rays in standing position. Patient 1 has a $50^{\circ} \mathrm{Cobb}$ angle, is $153.4 \mathrm{~cm}$ high, weighs $48.8 \mathrm{Kg}$, and a body mass index of 20.7. Patient 2 has a $49^{\circ}$ Cobb angle, is $160.5 \mathrm{~cm}$ high, weighs 46.2 .8 $\mathrm{Kg}$, and has a body mass index of 17.9. For each patient, T1-weighted axial ${ }^{*}$ and sagittal ${ }^{\dagger}$ MRI slices are acquired for the entire torso. The 3D shape of the thoracic and lumbar vertebrae is manually segmented from the sagittal slices using TomoVision's SliceOmatic software, and 8 landmarks are placed on the left and right edges of the posterior, anterior, inferior and superior ends of the vertebral bodies. These landmarks are used in order to drive the articulated model registration. A 3D geometric model of the spine from X-ray data is then obtained. The latter is generated by extracting the 3D position of 6 vertebral landmarks from postero-anterior and lateral radiographs using an explicit calibration method. ${ }^{14}$ The $3 \mathrm{D}$ location of the landmarks is then used in order to map generic vertebral surface meshes onto the patient space. In that case, 6 landmarks are placed on the centers of the superior and inferior plates of the vertebral bodies, and below and above the left and right pedicles. In addition, radio-opaque markers are placed on the surface of the patient's torso and are then manually localised on the obtained X-rays. The 3D location of these surface landmarks is also obtained using the explicit calibration method. Finally, a mesh representation of the surface topography data is obtained by merging data from four 3D digitizers (Creaform inc., $1.1 \mathrm{~mm}$ resolution) obtained from different views. Prior to image acquisition, adhesive markers are placed on the surface of the patient's torso at the same location as for X-ray acquisition. These markers are then manually localised using in-house software, and their 3D coordinates are obtained.

Patient 1 is selected in order to construct a generic model that contains bone, soft tissue, and surface topography information. This model is obtained by performing intra-patient registration of the patient's MRI,

\footnotetext{
*(1mm by $1 \mathrm{~mm}$ in-plane resolution, $2 \mathrm{~mm}$ thickness and $12 \mathrm{~mm}$ spacing between slices. Siemens Symphony system 1.5 Tesla, $\mathrm{TR} / \mathrm{TE}=650 / 12,704 \times 704$ )

${ }^{\dagger}(1.5$ Tesla, $\mathrm{TR} / \mathrm{TE}=771 / 15,704 \mathrm{x} 704,350 \mathrm{FOV})$, with a $0.5 \mathrm{~mm}$ by $0.5 \mathrm{~mm}$ in-plane resolution and $3 \mathrm{~mm}$ thickness, with a $3.6 \mathrm{~mm}$ separation between slices
} 


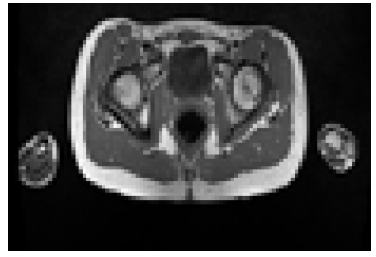

(a) Axial MRI slices

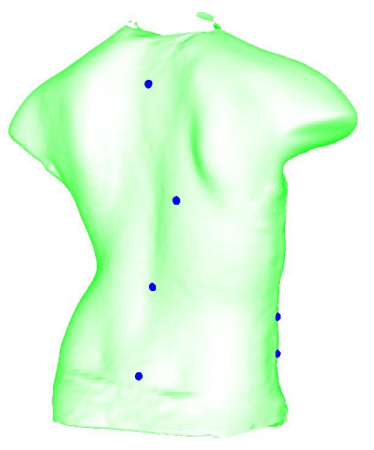

(b) Surface topography

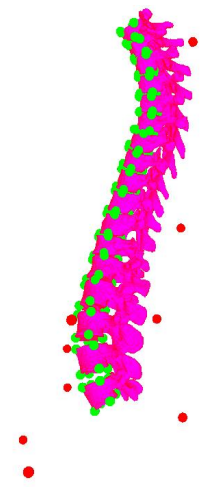

(c) Reconstructed X-ray

Figure 1. MRI, X-ray and surface topography data acquired for each of the 2 patients. Surface landmarks are displayed in blue of the surface topography and in red on the X-ray image. The vertebral landmarks used to construct the articulated model are displayed in green on the X-ray.

X-ray and TP data using articulated models and constrained thin-plate splines. ${ }^{3}$ The articulated model represents the spine as a series of rigid, local, inter-vertebral transformations. First, a local coordinate system is obtained for each of the lumbar and thoracic vertebrae in each of the MRI and X-ray modalities using the manually obtained vertebral landmarks. The rigid inter-vertebral transformations, $T_{v-1, v}$, between the local coordinate system of each vertebra $v$ and the local coordinate system of its lower neighboring vertebra $v-1$ are then obtained. Finally, the global transformation for each of the vertebrae in each of the image modalities is obtained using a composition of the local inter-vertebral transformations:

$$
T_{0, v}=T_{v-1, v} \circ T_{v-2, v-1} \circ \ldots \circ T_{1,2} \circ T_{0,1},
$$

where $T_{0,1}$ is the global rigid transformation between the world coordinates and the first vertebra on each of the two image modalities. The transformation $\left(T_{M R I-b o n e}\right)$ from MRI to X-ray space for each vertebra is then obtained by concatenating the global MRI and X-ray vertebral transformations. Finally, each axial MRI slice containing the soft tissues is registered using the articulated model transformation of the closest vertebra, followed by a thin-plate spline transformation that is weighted in such a way that its effect decreases the closer we get to the vertebra.

Once the generic model is obtained, patient 2 is selected as a test patient. A test patient model is obtained by incorporating the soft tissue information of the generic model to the X-ray and TP information of the test patient. A partial model of the test patient is first obtained by registering the TP and the X-ray reconstructions by using a thin-plate spline transform using the landmarks that were placed on the surface of the patient prior to data acquisition. Then, each axial MRI slice of the generic model is registered to the test patient's partial model in the following manner: First, the transformation $T_{v-M R I_{\text {model }}-X-\text { raypatient }}$ is obtained between each vertebra $v$ of the spinal reconstruction of the generic model's MRIs in prone position and the test patient's X-rays in standing position. This is done by concatenating the vertebral X-ray and MRI transformations:

$$
T_{v-M R I_{\text {model }}-X-\text { raypatient }}=T_{0, v-X-\text { raypatient }} \circ T_{0, v-M R I_{\text {model }}}^{-1}
$$

where $T_{0, v-M R I_{\text {model }}}^{-1}$ transforms the vertebra $v$ obtained from the MRIs of the generic model to the origin and

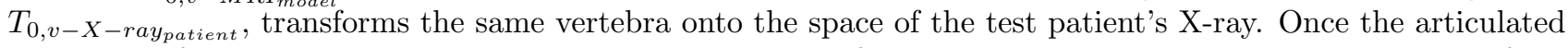
model transformations are obtained, each MRI slice is transformed using $T_{M R I}$. The latter is composed of the articulated model transformation $T_{v_{s}-M R I-b o n e}$ of the vertebra $v_{s}$ which is closest to the slice being registered and a nonrigid transformation $T_{M R I-s o f t_{\text {model-to-patient }}}$ which deforms the soft tissues such that they are contained within the space confined by the vertebral surfaces and the external surface of the test patient's torso. This transformation for each point $p$ of the MRI consists of a weighted thin-plate spline. First, a thin-plate spline is 
obtained using correspondence points on the contours of the MRI of the generic model and the contours of the surface topography of the test patient. This thin-plate spline is then weighted depending on the distance from the point to be deformed and the closest vertebra, in such a way that the transformation becomes more rigid as the point approaches the vertebra and less rigid as the point approaches the surface of the torso.

\section{RESULTS}

Figure 2 shows a result of the inter-patient registration between the generic model and the test patient in (a) and (b) compared to the intra-patient registration of the test patient in (c) and (d). When comparing the 2 registrations in (a) and (c), the slice is projected to the same vertebral level and has the same inclination in both cases. Thus, the articulated model successfully maps the MRI slice of the generic model to the right location in the test patient. When observing the quality of the deformable registration in Figures 2(c) and 2(d), the MRIs are well confined in the space between the vertebrae and the surface topography both following intra and inter-patient registration. Though there are intensity dissimilarities between the two MRIs, there is similarity in terms of the position, orientation, and shape of soft tissues such as fat (bright white outer layer of the MRI) and muscle (darker grey just inwards of the fat). Since the simulator under development only requires the modeling of the contours of anatomical structures, these similarities are deemed sufficient for our application.

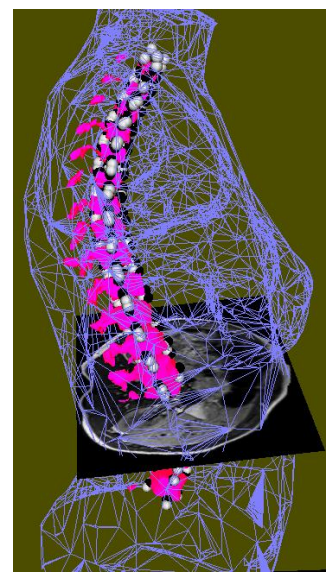

(a) Inter-patient registration from generic model

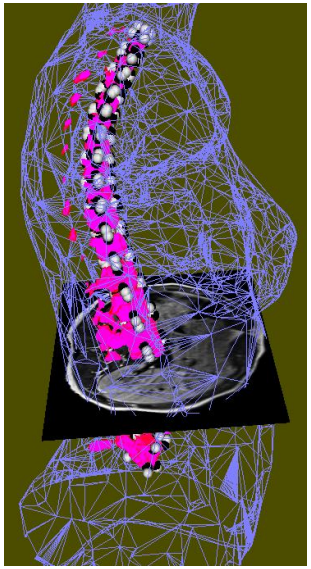

(c) Intra-patient registration of test patient

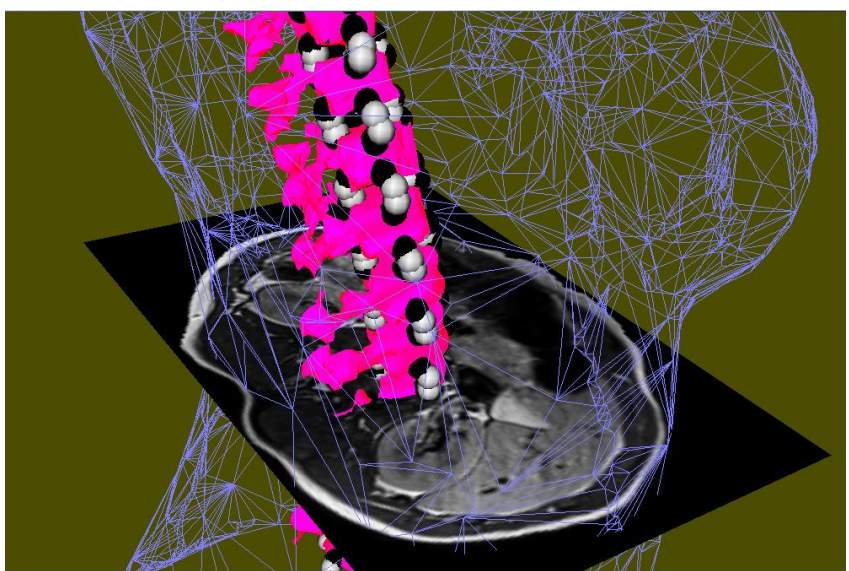

(b) Zoom of inter-patient registration from generic model

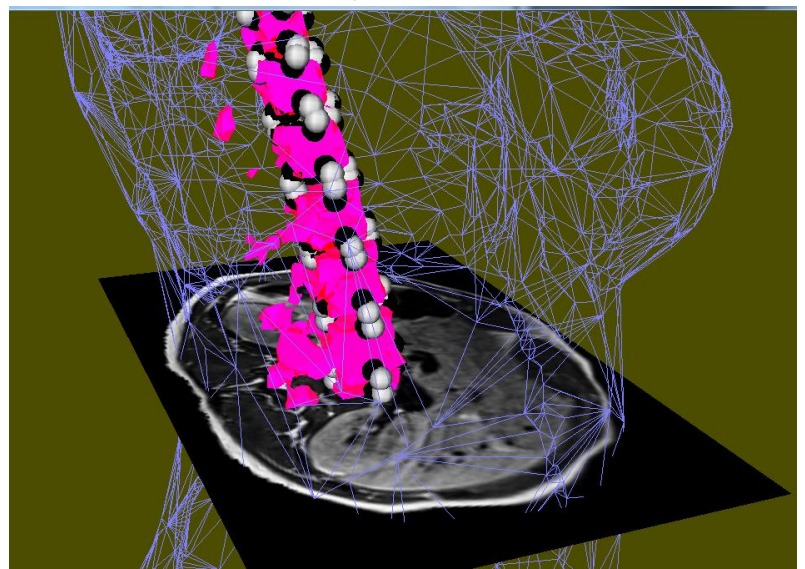

(d) Zoom of intra-patient registration of test patient

Figure 2. Inter-patient registration results between the generic model the the test patient shown for one MRI slice . 
Dice overlap values between the MRIs of the generic model following inter-patient registration and the TP of the test patient are shown in Figure 3. The average values are $0.898 \pm 0.013$ and $0.975 \pm 0.012$ for the rigid and proposed registration, respectively. A statistically significant improvement is observed when the proposed registration is used compared to rigid registration $(p<0.01)$. The inter-patient results are compared to those previously obtained following intra-patient registration of the test patient. The values obtained are very similar in both cases, where the average intra-patient registration Dice value is $0.976 \pm 0.009$ (Figure 4). The high overlap obtained following inter-patient registration is in large part due to the high similarity in the shape of the spine between the generic model and the patient. The similarities can be seen in Figure 5, where the local vertebral axes obtained from the MRI and X-ray data are shown for each of the 2 patients. As a result, the inter-vertebral transformations will be similar in both cases.

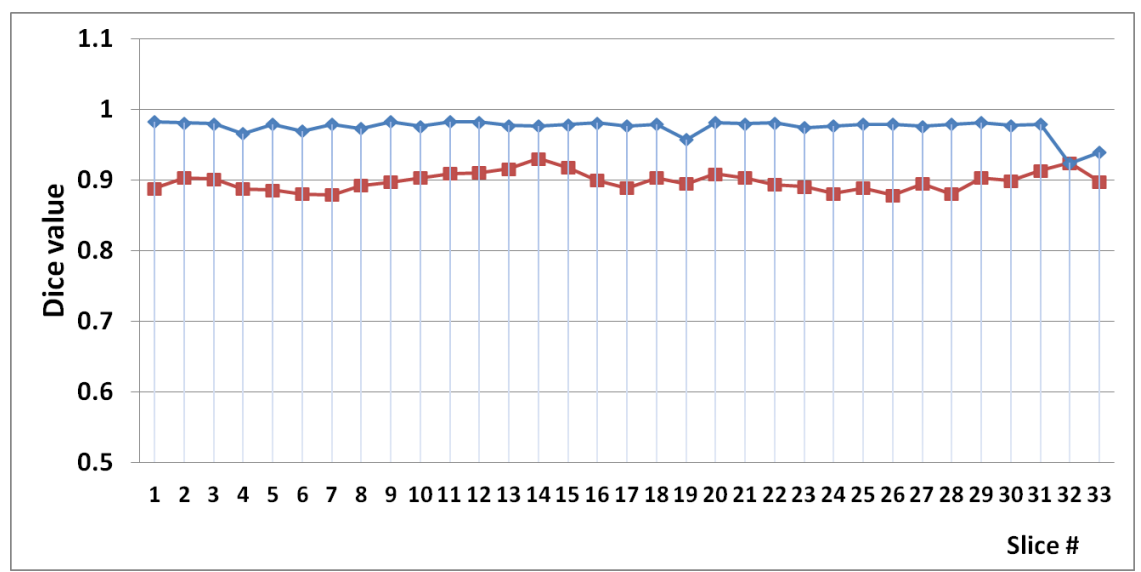

Figure 3. Dice values between the trunk surface of the test patient and the MRIs of the generic model following interpatient registration between the generic model and the test patient. The results obtained with the proposed method (blue) are compared to rigid registration (red).

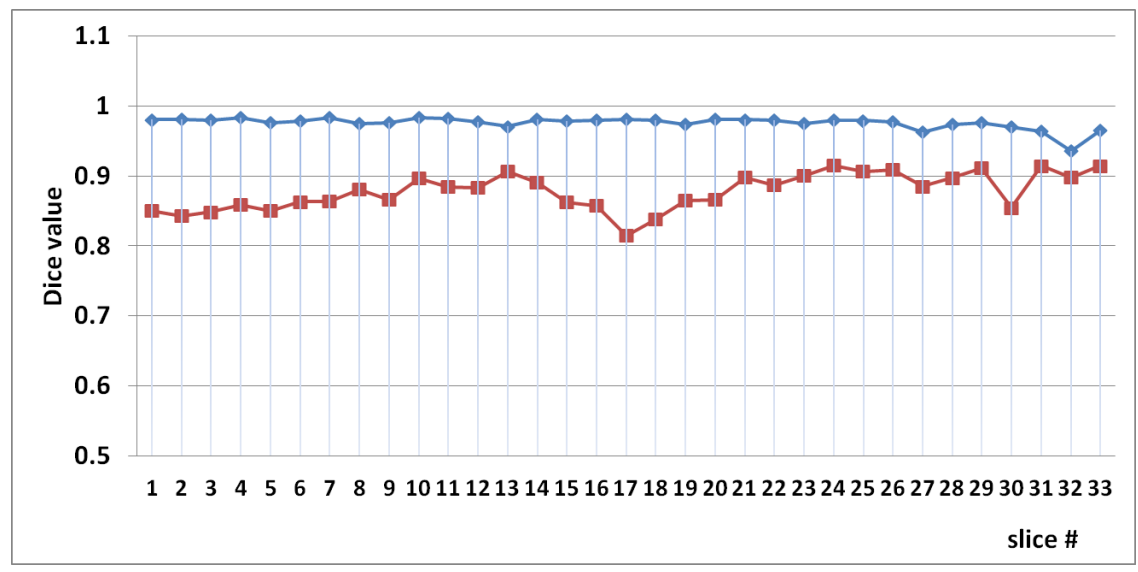

Figure 4. Dice values between the trunk surface and the MRIs of thetest patient following intra-patient registration of the test patient. The results obtained with the proposed method (blue) are compared to rigid (red) registration.

In order to assess the level of differences between the MRIs of the model and the test patient, the overlap is calculated between the MRIs of the generic model following inter-patient registration and the MRIs of the test patient following intra-patient registration. The resulting Dice values are shown in Figure 6. An average Dice value of $0.771 \pm 0.055$ is obtained after rigid registration. An average Dice value of $0.813 \pm 0.0833$ is obtained using the proposed registration method, showing greater similarity between the 2 MRIs following inter-patient registration compared to rigid registration. These values are lower than the Dice values obtained between the registered MRIs and the surface topography of the test patient. The decrease in overlap is evident in the upper 


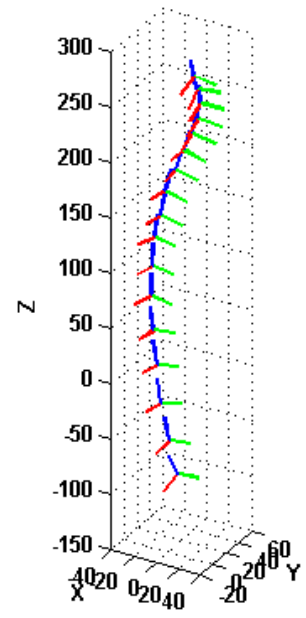

(a) MRI generic model

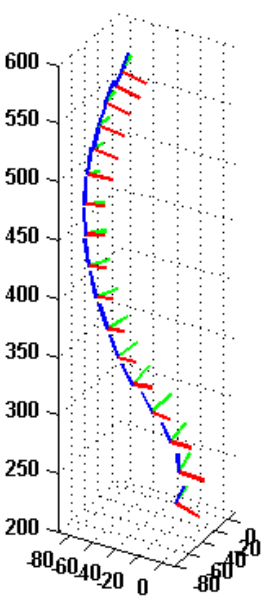

(b) X-ray generic model

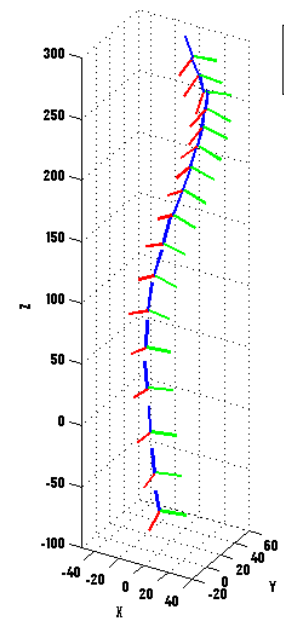

(c) MRI test patient

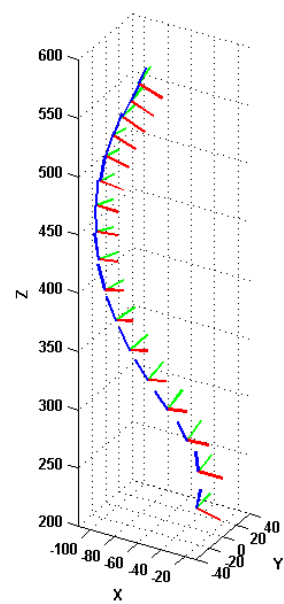

(d) X-ray test patient

Figure 5. Comparison of the vertebral axes obtained from the articulated model for the generic model and the test patient.

slices of the torso, particularly at the level of the shoulders. This is due to the difference in the rib hump between the 2 patients, which is not modeled using the articulated model. Nonetheless, the average Dice values obtained comparing the MRIs following inter-patient registration to the MRIs obtained using intra-patient registration of the test patient are significantly higher when the proposed method is used compared to rigid registration.

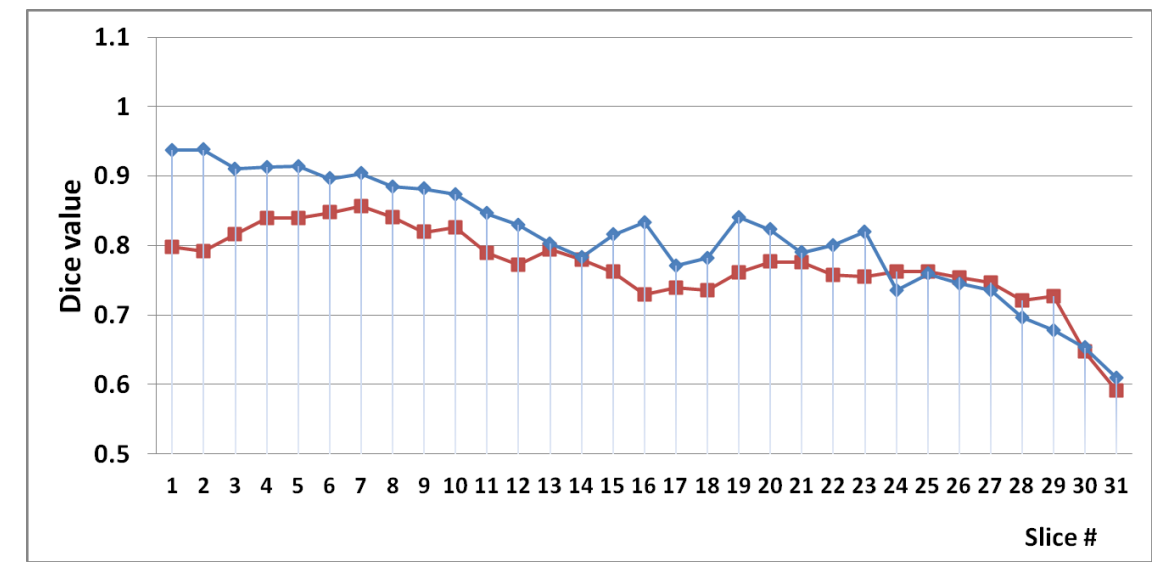

Figure 6. Dice values between MRIs of the generic model following inter-patient registration and the MRIs of the test patient following intra-patient registration. The results obtained with the proposed method (blue) are compared to rigid registration (red).

\section{CONCLUSION}

A method to obtain a full model incorporating bone structures, soft tissues, and the external surface of a scoliotic torso for surgical simulation was proposed. The model is obtained following inter-patient registration of MRI data from a generic model to TP and X-ray data of a test patient, as MRI data cannot always be acquired for patients in clinical settings. The MRIs from the generic model are fit into a partial patient model using articulated transformations in order to take into account the changes in patient posture between the image acquisitions, and thin-plate splines constrained to maintain bone rigidity and to fit in the space between the vertebrae and the external surface of the torso. The registration results obtained using the proposed method are comparable to previously obtained intra-patient registration results, and show a significant improvement 
compared to rigid registration. To our knowledge, this is the first works that proposes a method for multimodal inter-patient registration of the torso. We are currently in the process of obtaining a larger cohort of patients for further testing. In particular, we aim to test the effect of variations in height, weight and spinal curvature between the generic model and the test patient on the quality of the inter-patient registration. Future work also consists of modelling tissue-specific non-rigid deformations. The method developed can be used in order to obtain a geometric model of a patient which includes soft tissue information obtained from MRI data and can be incorporated in a surgical simulator even if MRI data cannot always be acquired for the patient.

\section{ACKNOWLEDGMENTS}

We would like to thank NSERC (Natural Sciences and Engineering Research Council of Canada) and the MENTOR (Canadian Institutes Health Research program) for their funding of this work.

\section{REFERENCES}

[1] Roach, J., "Adolescent idiopathic scoliosis," Orthop Clin North Am. 30, 353-365 (1999).

[2] Dionne, O. et al., "Simulation of the postoperative trunk appearance in scoliosis surgery," in [International Symposium on Biomedical Imaging (ISBI)], 1208 - 1211, IEEE, Newark, NJ (2012).

[3] Harmouche, R., Cheriet, F., Labelle, H., and Dansereau, J., "Multimodal image registration of the scoliotic torso for surgical planning.," BMC Medical Imaging 13(1) (2013).

[4] Schnabel, J. A., Rueckert, D., Quist, M., Blackall, J. M., Castellano-Smith, A. D., Hartkens, T., Penney, G. P., Hall, W. A., Liu, H., Truwit, C. L., Gerritsen, F. A., Hill, D. L. G., and Hawkes, D. J., "A generic framework for non-rigid registration based on non-uniform multi-level free-form deformations," in [Proceedings of the 4th International Conference on Medical Image Computing and Computer-Assisted Intervention], MICCAI '01, 573-581, Springer-Verlag, London, UK, UK (2001).

[5] Davis, M., Khotanzad, A., Flamig, D., and Harms, S., " A physics-based coordinate transformation for 3-D image matching.," IEEE Transactions on Medical Imaging 16(3), 317-328 (1988).

[6] Christensen, G. E., Rabbitt, R. D., and Miller, M. I., "3D brain mapping using a deformable neuroanatomy," Physics in Medicine and Biology 39(3), 609 (1994).

[7] Tang, S. and Jiang, T., "Fast non-rigid medical image registration by fluid model," in [Asian Conference on Computer Vision], 27-30 (2004).

[8] D'Agostino, E., Maes, F., Vandermeulen, D., and Suetens, P., "A viscous fluid model for multimodal nonrigid image registration using mutual information," Medical Image Analysis 7(4), 565 - 575 (2003).

[9] Bro-Nielsen, M. and Gramkow, C., "Fast fluid registration of medical images," in [SPIE Visualization in Biomedical Computing], Hone, K. and Kikinis, R., eds., 1131, 267-276, lncs (1996).

[10] Glocker, B. et al., "Non-rigid Registration using Discrete MRFs: Application to Thoracic CT Images," in [Workshop Evaluation of Methods for Pulmonary Image Registration, MICCAI], (2010).

[11] Tang, T. W. H. and Chung, A. C. S., "Non-rigid image registration using graph-cuts," in [Proceedings of the 10th international conference on Medical image computing and computer-assisted intervention - Volume Part I], MICCAI'07, 916-924, Springer-Verlag, Berlin, Heidelberg (2007).

[12] So, R. W. K., Tang, T. W. H., and Chung, A. C. S., "Non-rigid image registration of brain magnetic resonance images using graph-cuts," Pattern Recogn. 44, 2450-2467 (Oct. 2011).

[13] Xia, L. et al., "Automatic inter-subject registration of whole body images," in [Biomedical Image Registration. Proc. 3rd International Workshop, WBIR.], 18-25, Springer-Verlag, Utrecht, Netherlands (2006).

[14] Cheriet, F., Dansereau, J., Petit, Y., Aubin, C.-E., Labelle, H., and De Guisei, J., "Towards the selfcalibration of a multiview radiographic imaging system for the 3d reconstruction of the human spine and rib cage," International Journal of Pattern Recognition and Artificial Intelligence 13(5), 761-79 (1999). 